\title{
Diagnostic-therapeutic pathway for small lesions of the testis
}

\author{
Giulia Sbrollini ${ }^{1,2}$, Daniele Mazzaferro ${ }^{1}$, Asim Ettamimi ${ }^{1}$, Rodolfo Montironi ${ }^{3}$, \\ Marco Cordari ${ }^{1}$, Guevar Maselli ${ }^{1}$, Giacomo Tucci ${ }^{1}$, Gaetano Donatelli ${ }^{1}$, Francesco Pellegrinelli ${ }^{1,2}$, \\ Alessandro Conti ${ }^{1,2}$, Andrea Benedetto Galosi ${ }^{1}$ \\ ${ }^{1}$ Division of Urology, "Augusto Murri” Hospital, ASUR Marche, Fermo, Italy; \\ ${ }^{2}$ Institute of Urology, ${ }^{3}$ Institute of Pathology, Polytechnic University of the Marche Region, United Hospitals, Ancona, Italy.
}

\section{Summary Objective of our study was to define a diagnostic-therapeutic pathway for} proper treatment of not-palpable testicular masses, that may be benign in $38 \%$ of cases. Since the intraoperative diagnosis is difficult to reach in particular in small lesion $(<8 \mathrm{~mm})$ and the risk of tissue loss in frozen section analysis occurs frequently, we propose a diagnostic flow chart for the best management of small testis lesions. This proposed protocol has to be shown in details to physicians and patients, who must understand the clinical implications and the risk to undergo a second radical surgery.

KEY WORDS: Small masses; Testis neoplasms; Partial orchiectomy; Ultrasound; Pathology.

Submitted 3 October 2014; Accepted 31 October 2014

\section{INTRODUCTION}

Incidentally discovered small testicular lesions are increasing since ultrasound use increased in particular for infertility work up.

Although small lesion of the testis are benign in 38\% of cases $(1,2)$, there is no agreement on criteria for testissparing surgery.

Guidelines give indication for testis-sparing surgery only in selected cases (bilateral testicular tumor or monorchid patient) (3).

However, testis-sparing surgery has been performed also in patients with contralateral normal testis with a good oncological outcome $(2,4-8)$. Several concerns on conservative approach are debated: intraoperative diagnosis is not always reliable in relation to the small size of the lesion and also difficulties in pathological diagnosis related to the missing immunoistochemistry during intraoperative analysis.

The aim of this study is to establish a diagnostic-therapeutic pathway that allows to perform an effective testissparing surgery for impalpable testicular masses.
Clinical protocol to not-Palpable testis lesions We present a diagnostic-therapeutic protocol for patients affected by not-palpable testicular masses, with maximum diameter lower than $15 \mathrm{~mm}$ and negative testicular markers. This approach follows our clinical practice.

\section{Ultrasound}

All men underwent scrotal ultrasound in our hospital to confirm type, dimension and localization of the lesion. Ultrasound characteristics of the lesion were then verified at the confirmatory ultrasound by expert operator and last generation of ultrasound machine. If the lesion was not confirmed by confirmatory ultrasound or it is extra-testicular lesion, the patient was proposed for ultrasound follow-up.

Once the small testicular mass was confirmed at our hospital, the therapeutic indication for all cases was testicular exploration with inguinal access. This technique can be associated to intraoperative ultrasound, equipped with linear probe, in order to obtain the relative certainty of the size of the nodule and negative surgical margins, which of course will be subsequently verified by the pathologist. In figures, we report ultrasound images of three cases of our who underwent surgery for epidermoid cyst (Figure 2), Leydig tumor (Figure 3), and seminoma (Figure 4).

\footnotetext{
Surgery

Surgical exploration, using intraoperative ultrasound was done without clamping the spermatic cord. The surgical technique involved the removal of the neoplastic nodule and 3 additional biopsies of the surrounding parenchyma (two distant and one next to the mass) sent for definitive histology.

Smaller masses $(<8 \mathrm{~mm})$ were usually sent for definitive histology, while larger masses $(8-15 \mathrm{~mm})$ were sent to the pathologist for intraoperative frozen sections. Pathologist confirmed size and completeness of surgical margins, by macroscopic view. If the nodule was large enough to be cut for frozen section, then a microscopic description of malignant pattern was reported.
}

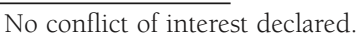


Figure 1.

Flow chart of diagnostic-therapeutic protocol for the management of non-palpable testicular masses.

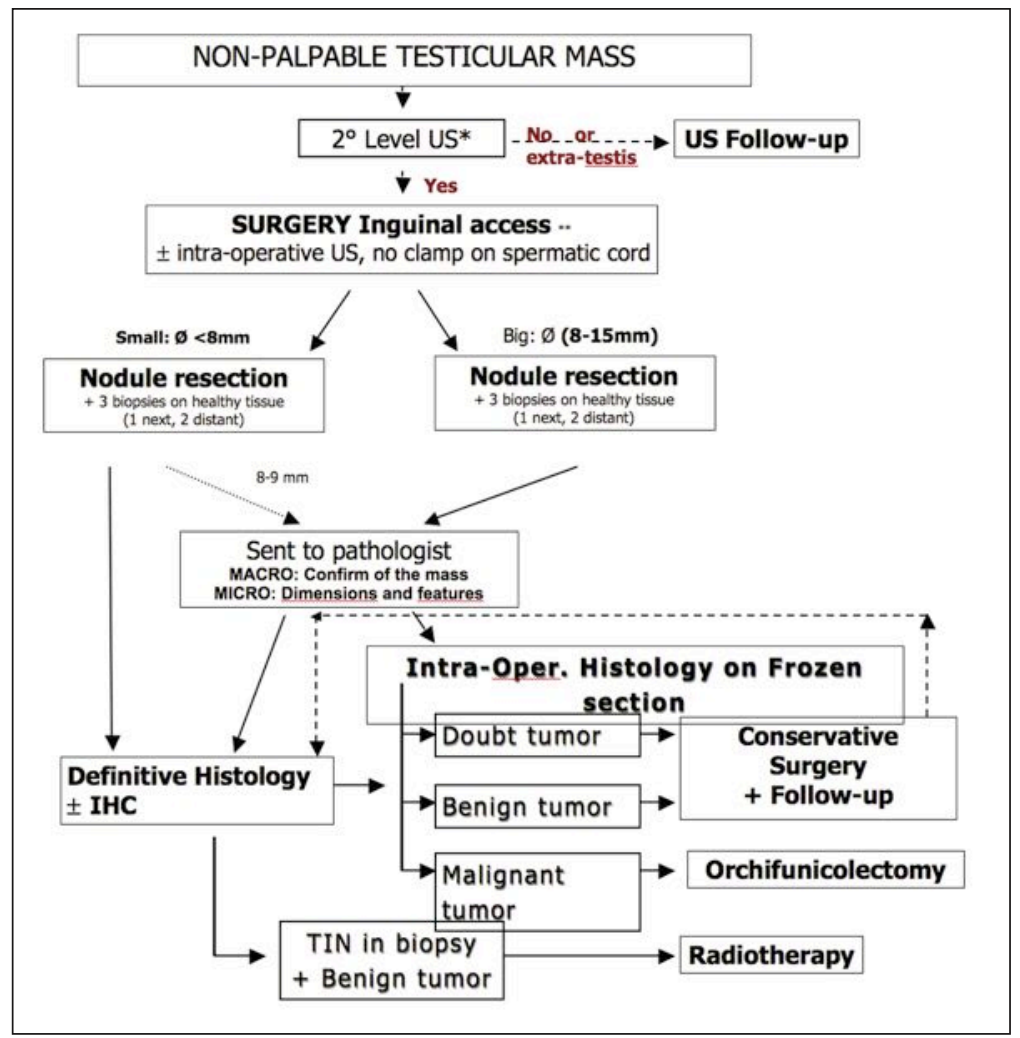

\section{Figure 2.}

Testis ultrasound: hyper-echoic lesion, not palpable, inside the testis. After surgical partial orchiectomy, a dermoid cyst was described.

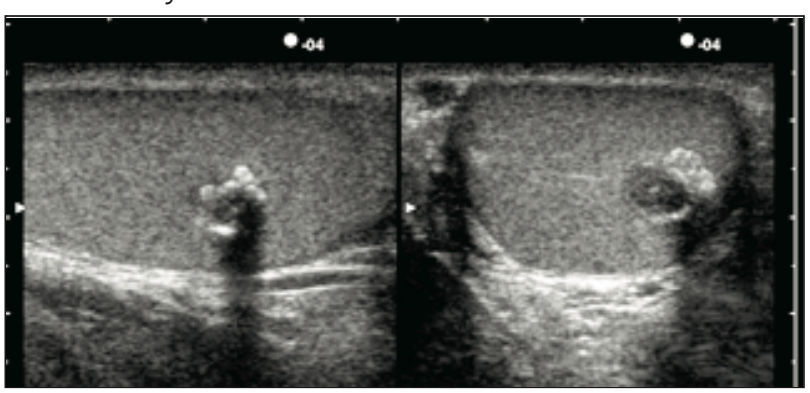

Figure 3

Testis ultrasound: small non palpable hypo-echoic lesion inside the testis. A Leydig tumor was identified after partial resection and definitive histology.

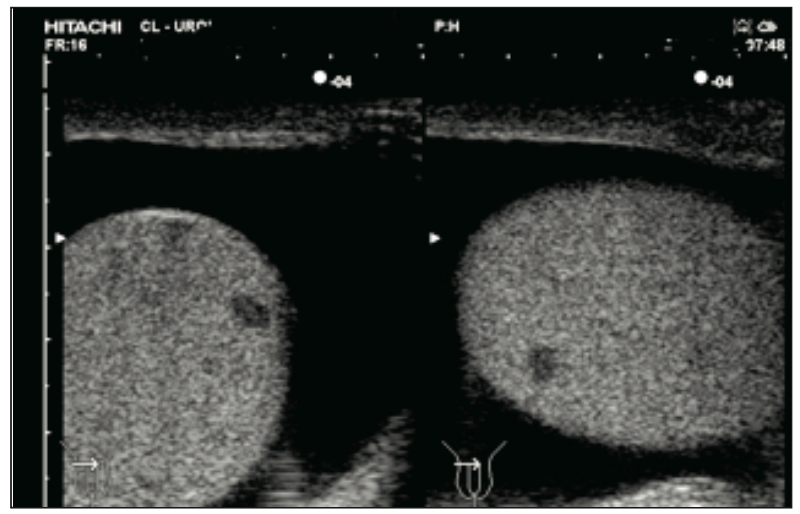

Masses of mean size (8-9 mm) were sent to the pathologist, who evaluated whether to perform the frozen section or to move directly to the definitive exam, in order to reduce the risk of losing tissue for definitive histology and immunoistochemistry. If feasible, the intraoperative frozen section histology gives real time information about macroscopic (localization, surgical margins, size of the lesion) and microscopic features.

Such information can guide the surgical management.

In case of benign or doubtful lesion there was the indication to keep on conservative management.

In case of malignancy at the frozen section, inguinal orchiectomy was performed.

All removed testicular lesion are subjected to definitive histological examination, including immunohistochemistry of the surgical specimen, that allowed to have a definitive histologic diagnosis. Definitive paraffin-embedded tissue analysis is the main diagnostic tool for testicular cancer. If definitive histology confirmed that the mass was benign, the patient was submitted to periodic ultrasound follow up. In case of definitive malignancy inguinal orchiectomy was done.

The presence of isolated intraepithelial neoplasia (TIN) in one or more of the biopsies of the surrounding parenchyma, posed indication to radiotherapy.

Before undergoing this protocol, the patient has to be informed in detail about the various benefits, risks and implications: first of all the possibility of being subjected to a second surgery as soon as the urologist is in possession of the definitive histological result.

Our protocol has been approved by different specialists involved in the diagnosis and treatment (urologist, pathologist, radiologist and oncologist) and used in different Departments of Urology.

Figure 1 shows the flow chart of the protocol for diagnosis, treatment and therapy of small not-palpable testicular masses.

\section{Figure 4}

Testis ultrasound: small non palpable hypo-echoic lesion close to the epididymis. A seminoma was identified after partial resection and definitive histology.

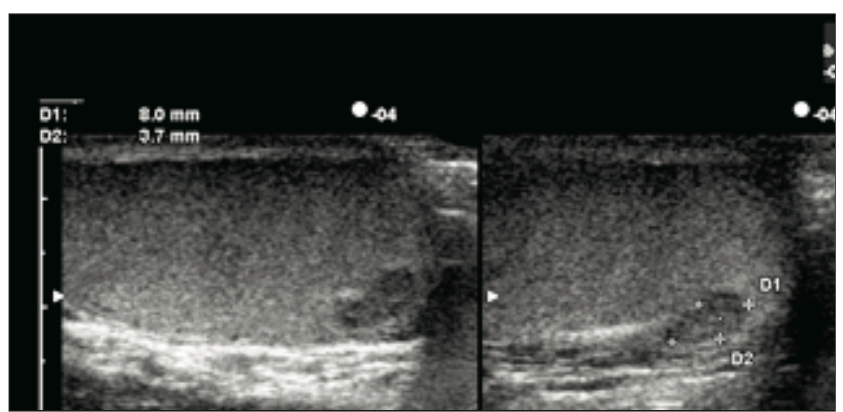


The histological diagnosis on frozen sections is difficult in small testicular masses and raises the risk of loss of tissue useful for the final histology and ancillary immunohistochemistry.

Intraoperative ultrasound allows the surgeon to orient with more confidence in the surgical practice and then to decide whether to send the specimen for intraoperative histological examination or for definitive examination.

In the masses of border-line size a key role in this decision is covered by the pathologist, who must be able to evaluate the tumor volume and then to act in a way to have an definitive histologic diagnosis as accurate as possible.

\section{Conclusions}

Frequency of benign tumors is higher in small testicular masses. Intraoperative diagnosis of non-palpable testicular lesions with frozen sections is difficult in particular for very small lesions $(<8 \mathrm{~mm})$ also for the risk of loss of tissue for definitive histology and ancillary immunoistochemistry.

For these reasons, our protocol allows the surgeon to perform testis-sparing surgery in selected cases, reaching an oncologic out-come similar to standard surgery.

\section{REFERENCES}

1. Shilo Y, Zisman A, Raz O, et al. The predominance of benign histology in small testicular masses. Urol Oncol. 2012; 30:719-22.

2. Shilo Y, Zisman A, Raz O, et al. Testicular sparing surgery for small masses. Urol Oncol. 2012; 30:188-91.

3. Albers P, Albrecht W, Algaba F, et al. Guidelines on testicular cancer. Eur Urol. 2011; 60:304-19.

4. De Stefani S, Isgrò G, Varca V, et al. Microsurgical testis-sparing surgery in small testicular masses: seven years retrospective management and results. Urology 2012; 79:858-62.

5. Subik MK, Gordetsky J, Yao JL, et al. Frozen section assessment in testicular and paratesticular lesions suspicious for malignancy: its role in preventing unnecessary orchiectomy. Hum Pathol. 2012; 43:1514-9.

6. Muller T, Gozzi C, Akkad T, Pallwein L, et al. Management of incidental impalpable intratesticular masses of $<$ or $=5 \mathrm{~mm}$ in diameter. BJU Int. 2006; 98:1001-4.

7. Gentile G, Brunocilla E, Franceschelli A, Schiavina R, et al. Can testis-sparing surgery for small testicular masses be considered a valid alternative to radical orchiectomy? A prospective single-center study. Clin Genitourin Cancer. 2013; 11:522-6.

8. Brunocilla E, Gentile G, Schiavina R, et al. Testis-sparing surgery for the conservative management of small testicular masses: an update. Anticancer Res. 2013; 33:5205-10.

9. Galosi AB, Lacetera V, Muzzonigro G. Clinica delle malattie testicolari di interesse ecografico. Urologia 2008; 75S12:559-66.

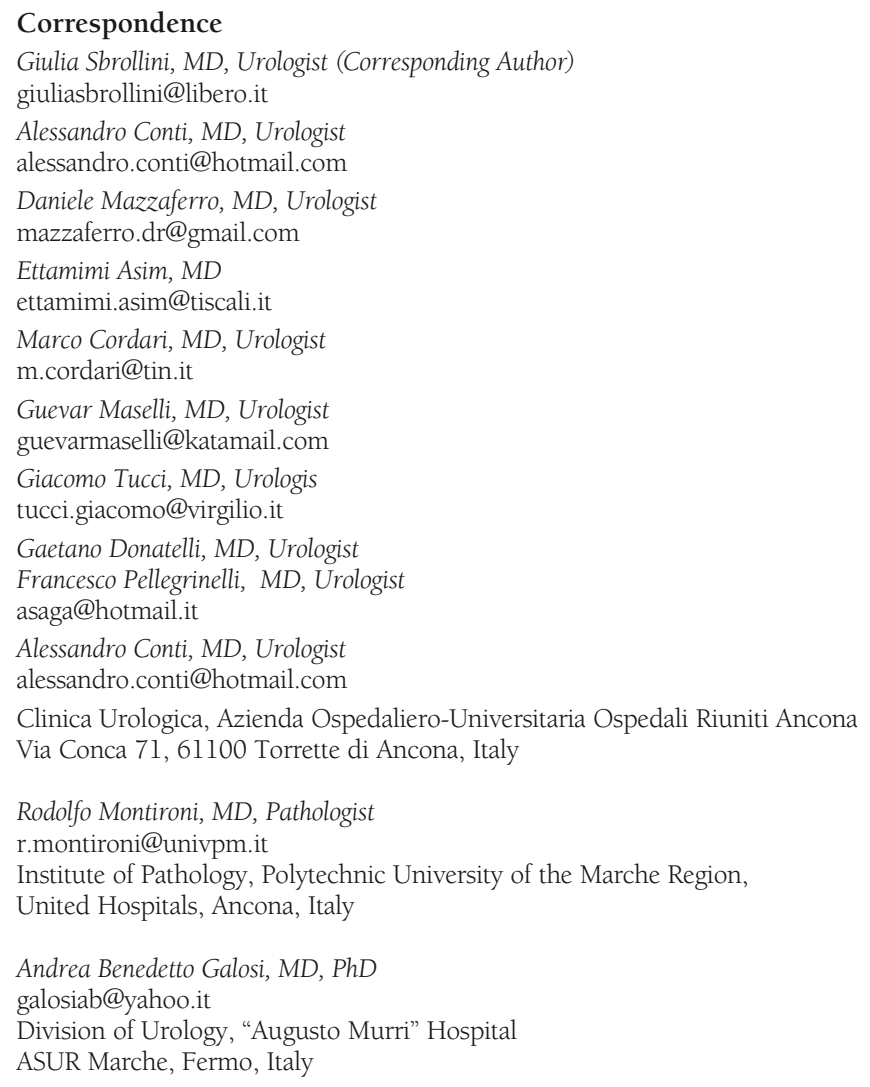

